\title{
Retrospective Qualitative and Quantitative Analysis of Adverse Events following Vaccination
}

\author{
Hardik Patel ${ }^{1}$, Samidh Shah ${ }^{2}$, Mira Desai ${ }^{3}$, V Kalaiselvan ${ }^{4}$, G.N Singh ${ }^{5}$ \\ 'Tutor, ${ }^{2}$ Assistant Professor, ${ }^{3}$ Professor and Head, Department of Pharmacology, B. J. Medical College, Ahmedabad- 380016, Gujarat, Uttar Pradesh, INDIA. \\ ${ }^{4}$ Principal Scientific Officer, Indian Pharmacopoeia Commission, Ministry of Health and Family Welfare (Govt. of India), Ghaziabad, INDIA, Uttar Pradesh. \\ ${ }^{5}$ Secretary-cum-Scientific Director, Indian Pharmacopoeia Commission, Ministry of Health and Family Welfare (Govt. of India), Ghaziabad, Uttar Pradesh, INDIA.
}

\begin{abstract}
Background: Vaccination is an essential component of the public health programs. A small minority of persons may experience mild, time limited adverse reactions, however, in rare cases, it may be serious. Hence there is a need of a surveillance program to monitor, record and analyze adverse event following immunization (AEFI). Method: All AEFIs in year 2015-2016 reported to NCC-PVPI were included in analysis. The data were entered in Excel sheet and analyzed for age group, gender, reporter detail, month wise reporting, seriousness, suspected vaccine and body system organ classification. Quality of AEFI was checked by completeness score as per WHO criteria. Result: The NCC-PvPI received 637 reports with 794 AEFIs. Majority of AEFIs were noted in infants $(55 \%)$ and the main source of AEFI reporters were physicians $(88 \%)$. The quality of reports were satisfactory with average completeness score 0.88. Category "body as a whole" (48.49\%) was the commonest clinical manifestation and most common AEFI was fever (43.95\%). DPT/Haemophilus influenza type B/Polio vaccine was most common causal vaccine $(n=155)$. Approximately $13 \%$ AEFIs were serious
\end{abstract}

in nature with seven deaths. Conclusion: The system of AEFI reporting in India is slowly gaining momentum. Majority of AEFIs were mild. However, serious AEFI needs detail reporting, follow up, investigation for causality assessment and identify risk factors. Organized training of health care professionals in AEFI reporting and targeted spontaneous reporting of vaccine AEFI will be helpful in this regard.

Key words: Adverse event following immunization (AEFI), Vaccine, Pharmacovigilance.

Correspondence

Dr. Samidh Shah, Assistant Professor, Department of Pharmacology, B. J. Medical College, Ahmedabad- 380016, Gujarat, INDIA.

Phone: 9825507413

Email: samidhshah@yahoo.com

DOI: 10.5530/jyp.2018.10.72

\section{INTRODUCTION}

Vaccination is an essential component of public health programme to save lives and preserves health. Unlike medicines, the expectations from vaccinations are much higher and problems arising from the vaccine or vaccination are less acceptable to the general public. ${ }^{1}$ However, like medicinal products, vaccines are not free from adverse events while it help people stay healthy. ${ }^{1}$ A small minority of persons may experience mild, time limited adverse reactions, however, in rare cases, it may be serious. $^{2}$

Adverse event following immunization (AEFI) is defined as any untoward medical occurrence which follows immunization and does not necessarily have a causal relationship with the usage of the vaccine. The adverse event may be any unfavourable or unintended sign, abnormal laboratory finding, symptom or disease. ${ }^{3}$ The aims of AEFI surveillance is to monitor immunization safety, detect and respond to adverse events; correct unsafe immunization practices, reduce the negative impact of the event on health and contribute to the quality of immunization activities. ${ }^{4}$

Studies conducted in Spain and USA showed that AEFI rate varies between $11.9-19 \%$ per 1000 doses. $^{4-5}$ Surprisingly in India, 54 deaths were reported by Pentavalent vaccine in $2014 .{ }^{6}$ Among them, three deaths were causally associated with vaccination, while one death has been placed under "Vaccine Reaction" by AEFI. ${ }^{6}$ According to recent media report up to August 2016 there have been 237 deaths reported within $72 \mathrm{~h}$ of vaccination with Pentavalent vaccine. ${ }^{7}$ A questionable relationship was reported earlier between measles, mumps and rubella (MMR) vaccine and autism, however recent report showed no association. ${ }^{8-9}$
One case series also reported an increase in the number of BCG induced lymphadenitis in India. ${ }^{10}$

In India, to monitor the safety of vaccines, Pharmacovigilance Programme of India (PvPI) has been coordinating with immunization division of Ministry of Health and Family Welfare, Government of India under AEFI program since 2013. The PvPI shares AEFI data reported from ADR monitoring centers (AMCs) across India, with the Immunization Technical Support Unit (ITSU) and AEFI- CDSCO. ${ }^{11}$ An attempt has been made in the present study to analyze AEFI data with respect to causal vaccine and system organ involved.

\section{METHODS}

This was a retrospective observational study carried out at PvPI Regional Training Center, Department of Pharmacology, B. J. Medical College, Ahmedabad. Ethics committee approval was obtained. The PvPI received AEFI data from ADR monitoring center (AMCs) across India. The data was collected from Pharmacovigilance Programme of India (PvPI) center, Ghaziabad. The data were entered in Excel sheet and analyzed for age group, gender, reporter detail, month wise reporting, seriousness, suspected vaccine and body system organ classification. An AEFI resulting into death, is life-threatening, requires in-patient hospitalization or prolongation of existing hospitalization, persistent or significant disability/ incapacity, or is a congenital anomaly/birth defect was considered serious. ${ }^{5}$ Quality of AEFI was checked by completeness score as per WHO criteria.? In addition, literature search using Google Search engine and Pubmed for original research articles/case reports to find out vaccine related 
adverse events in India or other countries. Comparison between completeness score of serious and non-serious AEFIs was done by unpaired $t$-test and $p$-value less than 0.05 was considered as significant.

\section{RESULTS}

A total of 637 AEFI reports consist of 794 events from various AMCs, health professionals, pharmacists and consumers during the study were reported.

\section{Age and Gender}

Out of 637 reports, majority $(353,55.42 \%)$ were observed in $0-12$ months of age group followed by $1-5$ years $(198,31.08 \%)$ [Figure 1]. However, in 18 reports no information on age was available. Majority of AEFI (343, $54 \%$ ) were reported in male while $270(42 \%)$ in female with male to female ratio of 1.27: 1 . However, in $24(3.77 \%)$ reports, gender information was unavailable.

\section{Month wise distribution and source of reports}

Interestingly, month wise distribution of the reports showed that it was well sustained throughout the year with maximum reports (112) in June 2015. In addition, majority of AEFI were reported by physicians (563, $88 \%)$, followed by the other healthcare professionals $(46,7 \%)$, pharmacists $(17,3 \%)$ and consumers or other non-healthcare professionals $(5,1 \%)$.

\section{Clinical presentation and body system affected}

The most common adverse event was fever $(349,43.95 \%)$ followed by injection site reactions $(101,12.72 \%)$, diarrhea $(55,6.93 \%)$, pharyngitis (42, 5.29\%), convulsion $(17,2.14 \%)$ and excessive crying $(16,2.02 \%)$. System Organ Class (SOC) classification using WHO Adverse Reaction Terminology (WHO-ART) showed that body as a whole $(385,48.49 \%)$ was most common system involved followed by application site disorders $(108,13.60 \%)$ [Table 1].

\section{Causal vaccine}

Pentavalent vaccine (DPT/Haemophilus influenza type B/Polio) was the most common causal vaccine (155) followed by DPT/Haemophilus influenza type B/Hepatitis B vaccine (91) [Table 2]. Out of 637 AEFI, 108 (16.95\%) were serious in nature. Among serious AEFIs, 73(67.59\%) required caused/prolonged hospitalization, 9 were life threatening and 7 were fatal. Seven deaths were temporally associated with HPV, Japanese encephalitis, DPT, hepatitis B, polio, measles, BCG and Haemophilus influenza type B vaccines. Interestingly, out of 7 fatal cases, 5 patients received multiple vaccines concurrently such as DPT (3), hepatitis B (3), polio (3), BCG (2), Haemophilus influenza type B (2) and measles (1) vaccines. While single fatal serious adverse event (SAE) was temporally associated with HPV and Japanese encephalitis each.

Table 1: System Organ Class (SOC) of adverse events following immunization $(n=794)$

\begin{tabular}{cc}
\hline System Organ Involved & Number (\%) \\
\hline Body as a whole & $385(48.49)$ \\
Application site disorders & $108(13.60)$ \\
Gastrointestinal disorders & $77(9.70)$ \\
Neurological disorders & $72(9.07)$ \\
Respiratory disorders & $62(7.81)$ \\
Skin and appendages disorders & $27(3.40)$ \\
Musculoskeletal disorders & $13(1.64)$ \\
Secondary terms - events Body as a whole - general disorders & $11(1.39)$ \\
Others & $39(4.91)$ \\
\hline
\end{tabular}

Table 2: Top ten causal vaccines for adverse events following immunization reports $(n=794)$

\begin{tabular}{cc}
\hline Causal Vaccines & Number \\
\hline DPT/Haemophilus influenza type B/Polio & 155 \\
DPT/Haemophilus influenza type B/Hepatitis B & 91 \\
DPT & 65 \\
MMR & 51 \\
DPT/Haemophilus influenza type B/Polio/rotavirus & 27 \\
Hepatitis A & 24 \\
Japanese encephalitis & 22 \\
Measles & 22 \\
DPT/Haemophilus influenza type B & 20 \\
BCG & 20 \\
Others & 297 \\
\hline
\end{tabular}

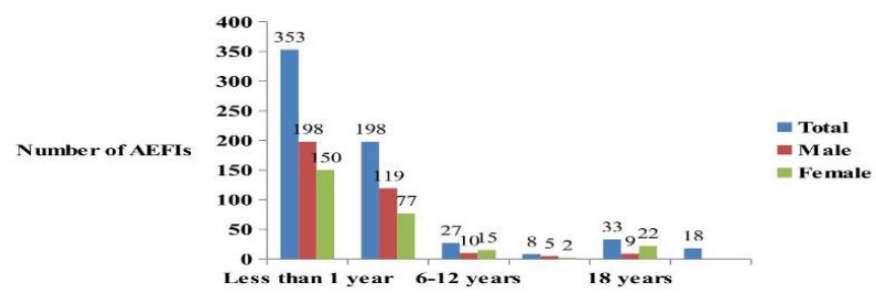

* Information not available

Figure 1: Age and Gender wise distribution of AEFI reports $(n=637)$

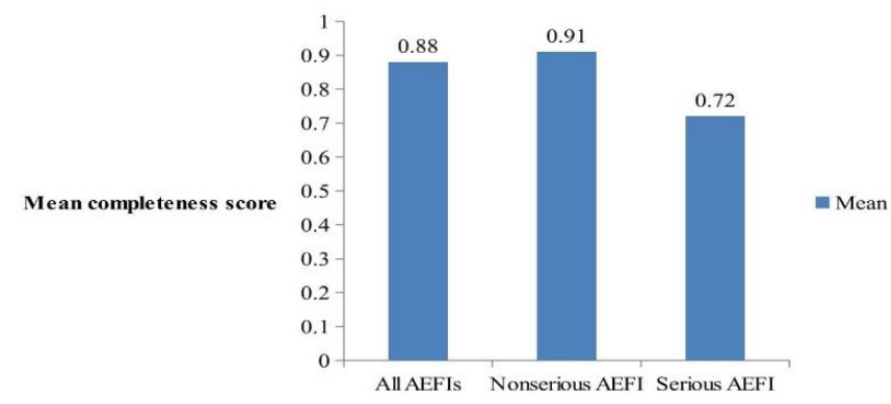

" $p<0.001$ as compared to non-serious AEFIs

Figure 2: Comparison of completeness score of non-serious AEFI versus serious AEFI reports $(n=637)$

\section{Completeness score}

The mean completeness score of AEFIs in study was 0.88. However, significant reduction in completeness score was observed in serious AEFI compared to non-serious reports by unpaired $t$-test $(p<0.001)$ [Figure 2].

\section{DISCUSSION}

Our analysis showed that majority of AEFIs were noted in infants and the main reporters were physicians. Fever was the commonest clinical manifestation and DPT/Haemophilus influenza type B/Polio vaccine was most common causal vaccine. Few AEFI reports were serious in nature with seven deaths.

A study by Joshi $\mathrm{N}$ et al. 2012 in India and Yu Hu et al. 2012 in China has reported $94.7 \%$ and $85.2 \%$ of AEFI in infants respectively. ${ }^{12-13}$ The 
adverse event reporting rate in infants in our study is less than above studies. The difference can be attributed to active surveillance methodology used for AEFI reporting. While our study adopted spontaneous reporting system (passive surveillance). Under-reporting is a well known fact in spontaneous reporting system as it depends upon the initiative and contribution from health care professionals. ${ }^{14}$

Male to female reporting of AEFI was similar to study done by Joshi et al. ${ }^{12}$ Male preponderance observed probably indicates extra care and importance of male child, seeking prompt medical treatment. ${ }^{15}$

In addition, our findings indicates that majority of AEFI has been reported by health care professional. A study by Kalaivani $\mathrm{M}$ et al. also showed that majority of AEFI (83.3\%) reported by physians. ${ }^{16}$ While consumer reporting in India is still in infancy as compared to developed countries like Australia. ${ }^{17}$ This indicates need of consumer awareness in adverse event reporting.

The most common clinical manifestation of adverse event was fever followed by injection site reaction. Our observations are similar to reports published by Joshi $\mathrm{N}$ et al. and Zhou et al. ${ }^{12-13}$ The common systems involved were body as a whole and administrative site reactions similar to study reported by Aagaard et al. ${ }^{18}$

The most common causal vaccine was DPT/Haemophilus influenza type B/Polio followed by DPT/Haemophilus influenza type B/Hepatitis B vaccine. While Joshi $\mathrm{N}$ et al. reported $\mathrm{DPT} / \mathrm{Hepatitis} \mathrm{B}$ were most common causal vaccine followed by BCG. ${ }^{12}$ A study by Mahajan et al. reports influenza, $\mathrm{H}_{1} \mathrm{~N}_{1}$ and DPT vaccines were commonly associated with AEFI in Australia. ${ }^{17}$ The difference can be attributed to immunization programme, extent and AEFI reporting trend of the country. Interestingly, high number AEFIs due to DPT vaccine has been attributed to high concentration of endotoxin. ${ }^{19}$

Almost $13 \%$ of AEFIs were serious in nature which is similar to a study done at USA. ${ }^{20}$ Total seven deaths were reported as temporally associated with vaccine administration. A study by Aagaard et al. in Denmark reports one-third AEFIs as serious with two deaths. ${ }^{16}$ Deaths after immunization are unfortunate and usually coincidental. Moreover, to categorize these fatal serious events as 'certain' has been a challenge for health care professionals, require complete information, detail investigation and follow up. Further, these serious AEFIs could also be due to administration error or programmatic error. In addition, few of the fatal case received three to four vaccines concurrently. However, it definitely warrants further research and post marketing surveillance to preserve public confidence in the immunization programme.

Completeness Score is a system to measure the amount of information provided on Individual Case Safety Reports (ICSRs). Higher completeness score increases authenticity of ICSR. According to Uppsala monitoring center, average completeness score of all ICSRs reported by India is around $0.90 .{ }^{8}$ Our study showed that average completeness score of all AEFIs was very close to national completeness score. However, completeness score of serious AEFI was significantly less as compared to all AEFI reports. This indicates that serious AEFI reports lack complete details. This is of paramount importance as complete information in serious AEFI helps in causality assessment, to identify the risk factors and subsequently signal generation. This indicates the need for organized training and skill development programme in AEFI reporting.

\section{Strengths of study}

Very few studies on vaccine safety monitoring in India have been published. It is one of the first kind of study where in volume of the data has been obtained from pan India. An attempt has been made to correlate completeness score and AEFI reporting. In addition, a detail analysis has been done which can help leads to certain conclusions.

\section{Limitations of Study}

Like any other study, there were also few limitations such as passive surveillance, lack of complete information, causality assessment and follow up. The authors do not have proportion of population received vaccine. Thus we were not able to calculate the incidence rate.

\section{CONCLUSION}

The system of AEFI reporting in India is slowly gaining momentum. Majority of AEFI is mild. However, serious AEFI needs detail reporting, follow up, investigation for causality assessment and identify risk factors. Organized training of health care professionals in AEFI reporting and targeted spontaneous reporting of vaccine AEFI will be helpful in this regard.

\section{CONFLICT OF INTEREST}

The authors declare no conflict of interest.

\section{ABBREVIATIONS}

AEFI: Adverse event following immunization; AMCs: ADR monitoring centers; ICSRs: Individual Case Safety Reports; ITSU: Immunization Technical Support Unit; PvPI: Pharmacovigilance Programme of India; SAE: Serious adverse event; SOC: System Organ Class.

\section{SUMMARY}

Vaccination is an essential component of the public health programs. Majority of AEFIs in our study were mild. However, some AEFIs were serious in nature. Organized training of health care professionals in AEFI reporting and targeted spontaneous reporting of vaccine AEFI will be helpful in this regard.

\section{REFERENCES}

1. Sebastian J, Parthasarathi G, Ravi MD. Is there a Role for Pharmacist in Safety Monitoring of Vaccines?. IJPER. 2018;52(2):202-6.

2. WHO. Global vaccine safety blueprint. Quality, Safety and Standards unit of the Department of Immunization, Vaccines and Biologicals. Switzerland. 2012. [Internet]. [Cited 2017 May12]. Available from whqlibdoc.who.int/hq/2012/ WHO_IVB_12.04_eng.pdf?ua=1.

3. Carrasco-Garrido P, Gallardo-Pino P, Jiménez-García $R$, et al. Incidence of adverse reactions to vaccines in a paediatric population. Clin Drug Invest. 2004;24(8):457-63.

4. Morales-Olivas FJ, Martinez-Mir I, Ferrer JM, et al. Adverse drug reactions in children reported by means of the yellow card in Spain. J Clin Epidemiol. 2000;53(10):1076-80.

5. Council for international organizations of medical sciences (CIOMS) c/o World Health Organization. Definition and Application of Terms for Vaccine Pharmacovigilance: Report of CIOMSNHO Working Group on Vaccine Pharmacovigilance. 2012. Accessed on 12 May 2017. Available from: http://www.cioms.ch/frame_ vaccine_pharmacovigilance.html.

6. Pharmacovigilance Programme of India (PvPI). Performance Report 2015-16. Ghaziabad: 2016. Last assesse. 2017. Available from http://ipc.nic.in/writereaddata/mainlinkFile/File675.pdf

7. The Uppsala Monitoring Centre. Documentation Grading - Completeness Score. Last assessed on May 12 2017. Available from http://ipc.nic.in/writereaddata/mainlinkFile/File252.pdf.

8. Eggertson I. Lancet retracts 12-year-old article linking autism to MMR vaccines CMAJ. 2010;4(182):199-200

9. Jain A, Marshall J, Buikema A, et al. Autism occurrence by MMR vaccine status among US children with older siblings with and without autism. JAMA. 2015; 313(15):1534-40.

10. Manju V, Parida A, Hegde A, Kousikakatakam P. Upsurge in BCG vaccine induced lymphadenitis: Case series. J Young Pharm. 2018;10(1):123-5.

11. Puliyel J. AEFI and the pentavalent vaccine: looking for a composite picture. IJME. 2013;10(3):142-46.

12. Joshi N, Prajapati $H$, Solanki K, et al. Pattern of Adverse Events Following Immunization in an indian teaching hospital. Int J Med Sci Public Health. 2013;2(1):62-5.

13. Yu Hu, Qian Li, Luoya Lin et al. Surveillance for Adverse Events following Immu- 
nization from 2008 to 2011 in Zhejiang Province, China. Clin. Vaccine Immunol. 2013;20(2):211-7.

14. Gupta S, Nayak R, Shivaranjani R, et al. Questionnaire study on the knowledge, attitude, and the practice of pharmacovigilance among the healthcare professionals in a teaching hospital in South India. Perspect Clin Res. 2015;6(1):45-52.

15. Karamata V, Gandhi A, Patel $P$ et al. A study of the use of drugs in patients suffering from psoriasis and their impact on quality of life. Indian J Pharmacol. 2017;49(1):84-8.

16. Kalaivani M, Rastogi S, Kalaiselvan V, Singh GN. Adverse Reactions after Hepatitis B Vaccination: A Retrospective Analysis Using Spontaneous Reports. J Young Pharm. 2017:9(1):55-9.

17. Mahajan D, Cook J, Dey A, et al. Annual report: Surveillance of adverse events following immunisation in australia 2011. Commun Dislntell. 2012;36(4):

\section{E315-E332.}

18. Aagaard L, Hansen EW, Hansen EH. Adverse events following immunization in children: Retrospective analysis of spontaneous reports over a decade. Eur $\mathrm{J}$ Clin Pharmacol. 2011;67(3):28-38.

19. Lloyd JC, Haber P, Mootrey GT, et al. Adverse event reporting rates following tetanus-diphtheria and tetanus toxoid vaccinations: Data from the Vaccine Adverse Event Reporting System (VAERS), 1991-1997. Vaccine. 2003;8(21):25-6.

20. Zhou W, Pool V, Iskander JK, English-Bullard R, Ball R, Wise RP, et al. Surveillance for safety after immunization: Vaccine Adverse Event Reporting System (VAERS) - United States, 1991-2001. MMWR Surveill Summ. 2003;52(1):1-24.

Article History: Submission Date : 14-02-2018; Revised Date : 09-04-2018; Acceptance Date : 07-05-2018.

Cite this article: Patel H, Shah S, Desai M, Kalaiselvan V, Singh GN. Retrospective Qualitative and Quantitative Analysis of Adverse Events Following Vaccination. J Young Pharm. 2018;10(3):326-9. 